\section{The hyperdense middle cerebral artery sign in a polycythaemic child}

To the Editor: The hyperdense middle cerebral artery (HMCA) sign is known to be an early sign of intracranial arterial occlusion or infarction during unenhanced CT imaging of the brain. ${ }^{1}$ This sign has also been seen after treatment with bromide, ${ }^{2}$ in cocaine abusers ${ }^{3}$ and in adults with elevated haematocrit, ${ }^{4}$ but has not previously been reported to be a result of polycythaemia in children. We recently encountered this sign as an incidental finding throughout the vessels of the circle of Willis in a child with a cyanotic heart condition and speculate that the dense appearance of these intracranial vessels was the result of polycythaemia. A 4-year-old girl was admitted with headaches, vomiting and fever. The child had an atrial septal defect, ventricular septal defect, pulmonary atresia and multiple aorto-pulmonary collateral arteries. Clinically, she was cyanotic and clubbed. The neurological examination was normal. Laboratory tests showed a haematocrit of $67.6 \%$ and haemoglobin level of $21.9 \mathrm{~g} / \mathrm{dl}$ in keeping with polycythaemia. A CT scan of the head was performed in
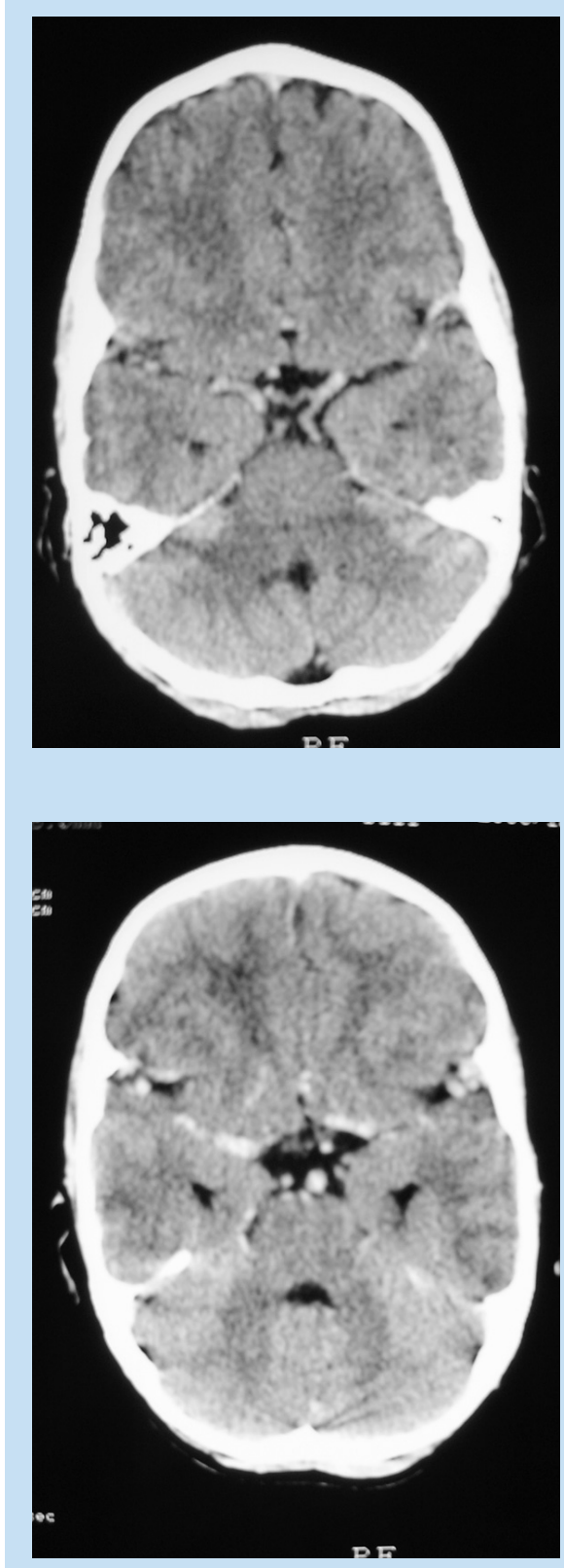

Figs $1 \mathrm{a}$ and $b$. Sequential non-contrast CT slices in a 4-year-old girl show increased density of the arteries of the circle of Willis due to polycythaemia. order to exclude a brain abscess or meningitis. The uncontrasted CT scan did not show any findings of a brain abscess but the HMCA sign was seen involving both middle cerebral arteries as well as the basilar artery (Figs $1 \mathrm{a}$ and $b$ ). There was no clinical or radiological evidence of cerebral infarction or vessel occlusion. The high haematocrit may therefore have increased the density of the blood leading to an HMCA sign. Although the HMCA sign has been shown to be a false indicator of vascular occlusion in adults, ${ }^{4}$ an association between an elevated haematocrit and a HMCA in children has not yet been reported.

\section{H Douis S Andronikou S Jadwat}

Department of Paediatric Radiology Red Cross Children's Hospital

University of Cape Town

\section{References}

1. Petitti N. The hyperdense middle cerebral artery sign. Radiology 1998; 208: 687-688.

2. Osborne D, Bohan T, Hodson A. CT demonstration of hyperdense cerebral vasculature due to bromide therapy. J Comput Assist Tomogr 1984; 8: 982-984.

3. Hall A, Wagle V. CT enhancement after use of cocaine. Am J Neuroradiol 1990; 11:1083.

4. Rauch RA, Bazan C 3rd, Larsson EM, Jinkins JR. Hyperdense middle cerebral arteries identified on CT as a false sign of vascular occlusion. Am J Neuroradiol 1993; 14: 669-673. 